\title{
openheart Association and diagnostic utility of diastolic dysfunction and myocardial fibrosis in patients with Fabry disease
}

Dan Liu, ${ }^{1,2}$ Daniel Oder, ${ }^{1,2}$ Tim Salinger, ${ }^{1,2}$ Kai Hu, ${ }^{1,2}$ Jonas Müntze, ${ }^{1,2}$ Frank Weidemann, ${ }^{2,3}$ Sebastian Herrmann, ${ }^{1,2}$ Georg Ertl, ${ }^{1,2}$ Christoph Wanner, ${ }^{1,2}$ Stefan Frantz,, ${ }^{1,2}$ Stefan Störk, ${ }^{1,2}$ Peter Nordbeck ${ }^{1,2}$

To cite: Liu D, Oder D, Salinger T, et al. Association and diagnostic utility of diastolic dysfunction and myocardial fibrosis in patients with Fabry disease. Open Heart 2018;5:e000803. doi:10.1136/ openhrt-2018-000803

DL, D0 and TS contributed equally.

Received 15 February 2018 Revised 21 May 2018

Accepted 13 June 2018
Check for updates

(C) Author(s) (or their employer(s)) 2018. Re-use permitted under CC BY-NC. No commercial re-use. See rights and permissions. Published by BMJ.

${ }^{1}$ Department of Internal Medicine I, University Hospital Würzburg, Würzburg, Germany ${ }^{2}$ Comprehensive Heart Failure Center (CHFC), University of Würzburg, Würzburg, Germany ${ }^{3}$ Medizinische Klinik I, Klinikum Vest, Recklinghausen, Germany

Correspondence to Dr Peter Nordbeck; nordbeck p@ukw.de

\section{ABSTRACT}

Objectives Current guidelines highlight important therapy implications of cardiac fibrosis in patients with Fabry disease (FD). However, association between morphological and functional impairments with cardiac fibrosis in hereditary cardiomyopathies remains elusive. We investigated the association between echocardiographydetermined cardiac dysfunction and cardiac MRI (cMRI)-detected myocardial fibrosis (late gadolinium enhancement, LE) in patients with FD with preserved left ventricular ejection fraction $(\geq 50 \%)$.

Methods 146 patients with FD (aged $39 \pm 14$ years, 57 men) were analysed, all receiving echocardiography and cMRI within a 1 week interval. Longitudinal systolic strain (LS_sys), strain rate (LSr_sys) and diastolic strain rate (LSr_E/LSr_A) were assessed using speckle-tracking imaging. Receiver operating characteristic (ROC) analysis was performed to identify the diagnostic performance of various markers for $\mathrm{LE}$

Results LE was detected in 57 (39\%) patients with FD. LV wall thickness, left atrial volume, septal E/e', diastolic dysfunction grade, global LS_sys and E/LSr_E, mid-lateral LS_sys and LSr_E, as well as N-terminal pro-brain natriuretic peptide were all associated with LE independent of age, sex, body mass index, New York Heart Association functional class and kidney function. In ROC curve analysis, septal E/e' performed best (area under the curve $=0.86,95 \% \mathrm{Cl}=0.79$ to 0.92 ). Septal $\mathrm{E} / \mathrm{e}^{\prime}>14.8$ was strongly associated with LE (specificity $=97.8 \%$ and sensitivity $=49.1 \%$ ). In $9 \%$ of patients, localised LE was present even though no other cardiac or kidney abnormalities were detected.

Conclusions Echocardiography-derived diastolic dysfunction is closely linked to LE in FD. Septal E/e' ratio is the best echocardiographic marker suggestive of LE. Diastolic dysfunction is not a prerequisite for $L E$ in FD, since LE can be detected in the absence of measurable cardiac functional impairments.

Trial registration number ClinicalTrials.gov Identifier (NCT03362164).

\section{INTRODUCTION}

Fabry disease (FD) is an X-linked multisystem disorder characterised by alpha-galactosidase A enzyme deficiency leading to progressive accumulation of globotriaosylceramide in

\section{Key questions}

What is already known about this subject?

- Myocardial fibrosis as detected by late gadolinium enhancement (LE) in cardiac MRI (CMRI) is associated with morphological and functional impairments including increased myocardial hypertrophy and myocardial stiffness.

- Enzyme replacement therapy is less effective in patients with Fabry disease (FD) already showing myocardial fibrosis.

What does this study add?

- Our results suggest that diastolic dysfunction is closely linked to LE in FD; however, cardiac involvement (LE) may also appear in some patients with FD in the absence of detectable systolic and diastolic dysfunction.

How might this impact on clinical practice?

- cMRI should be routinely applied to patients with FD to detect LE as potential first sign of cardiac involvement, implicating an indication for the initiation of specific therapy.

- The impact of enzyme replacement on LE in patients with FD without detectable systolic and diastolic dysfunction and renal dysfunction remains to be determined.

lysosome carrying cells. ${ }^{1}$ The occurrence of cardiomyopathy in FD is an ominous prognostic sign and today represents the leading cause of death in these patients. ${ }^{2-4}$ This cardiomyopathy is characterised by left ventricular (LV) hypertrophy, prominently hypertrophied papillary muscles, myocardial replacement fibrosis, ${ }^{5-8}$ right ventricular dysfunction $^{9}$ and rhythm disorders. ${ }^{10}$

Fibrotic replacement of myocardial fibres is another hallmark of disease progression in FD. Replacement fibrosis can be detected by cardiac MRI (cMRI) through visualisation of late gadolinium enhancement (LE). ${ }^{11}$ Experimental and clinical studies showed that the extent of LE is associated with increased 
myocardial stiffness and the degree of diastolic dysfunction in various cardiovascular diseases. ${ }^{12-15}$ Global systolic function often remains preserved in FD until its terminal stage; however, the development of more subtle alterations such as diastolic function frequently evades routine diagnostics. In particular, the association of diastolic dysfunction with myocardial replacement fibrosis in FD is not fully understood. Echocardiography plays a central role in the assessment of LV diastolic function. The diastolic strain rate measured by two-dimensional speckle-tracking imaging (STI) emerged as a feasible surrogate of LV filling pressure. For example, the ratio of early diastolic mitral inflow velocity to global early diastolic strain rate was particularly sensitive in detecting diastolic dysfunction in patients with acute myocardial infarction, superior to conventional diastolic indices. ${ }^{16}$

Although clinical studies demonstrated that specific enzyme replacement therapy (ERT) successfully alleviated the symptom burden in FD, established Fabry cardiomyopathy was generally poorly responsive. ${ }^{2}{ }^{17} 18$ Since treatment success critically depends on early therapy initiation, detection of subtle cardiac involvement might aid screening of patients with FD likely to benefit from early ERT.

We therefore investigated in patients with FD and preserved left ventricular ejection fraction (LVEF) the association between systolic/diastolic dysfunction as determined by echocardiography and myocardial fibrosis as detected by cMRI. We hypothesised that in these patients: (1) LE is linked not only to reduced longitudinal systolic strain (LS_sys) but also to diastolic dysfunction; (2) diastolic or systolic dysfunction is a prerequisite of replacement fibrosis indicated by LE; (3) STI-derived diastolic parameters might be superior to conventional diastolic indices for the staging of cardiac involvement.

\section{METHODS}

\section{Study population}

The database of the Würzburg Fabry Center for Interdisciplinary Treatment was searched for genetically proven patients with FD referred to the Center between 2006 and 2015. The structure of the center and the methodology of clinical phenotyping of patients with FD have been reported in detail elsewhere. ${ }^{19}$ All patients underwent both transthoracic echocardiography and cMRI within a 1 week interval. Out of 214 consecutive patients with FD, 165 had viable cMRI scans; 19 patients were excluded due to compromised LVEF $(n=9)$ or reasons promoting $\mathrm{LE}$ ( $\mathrm{n}=1$ diabetes; $\mathrm{n}=9$ coronary artery disease), rendering 146 patients with echocardiographically measured LVEF $\geq 50 \%$ suitable for the current analysis (online supplementary eFigure 1). The study was conducted in accordance to the Declaration of Helsinki. Written informed consent was obtained from all patients or their guardians. cMRI was performed using a 1.5 Tesla full-body MRI scanner (Magnetom Symphony Quantum/ Avanto; Siemens Medical Systems, Erlangen, Germany).
LE images were acquired 15 min after intravenous injection of $0.2 \mathrm{mmol} / \mathrm{kg}$ gadopentetate dimeglumine, using T1-weighted inversion recovery imaging sequences (field of view $240 \times 320 \mathrm{~mm}^{2}$, matrix size $165 \times 256$, slice thickness $8 \mathrm{~mm}$, echo time $3.4 \mathrm{~ms}$, repetition time $7.5 \mathrm{~ms}$ ). LE was rated by two radiologists as described before ${ }^{19}$ who were kept blinded to all clinical data. Areas with signal intensity above the average of the normal myocardium plus 2 SDs were defined as LE positive.

\section{Conventional echocardiography measurements}

A standard echocardiographic examination was performed on a Vivid 7 or 9e echo machine (GE Vingmed, Horten, Norway). Measurements were performed offline by a single investigator (DL) according to recommended guidelines as previously described. ${ }^{20}$ Pulsed-wave Doppler and tissue Doppler were recorded and diastolic parameters including mitral inflow peak early (E-wave) and late (A-wave) velocities, E/A ratio, deceleration time (DT) of $\mathrm{E}$ velocity and isovolumetric relaxation time (IVRT) were measured. ${ }^{21}$ Diastolic filling patterns (ie, normal, impaired relaxation, pseudonormal and restrictive filling) were defined according to current guidelines. ${ }^{21}$ In brief, a normal filling pattern was defined as mitral inflow $\mathrm{E} / \mathrm{A}$ ratio $=0.8$ to $2, \mathrm{DT}=140$ to $240 \mathrm{~ms}$, IVRT $=70$ to 100 $\mathrm{ms}$; impaired relaxation defined as the $\mathrm{E} / \mathrm{A}$ ratio $\leq 0.8$, DT $\geq 240 \mathrm{~ms}$, IVRT $>100 \mathrm{~ms}$; pseudonormal filling defined as the $\mathrm{E} / \mathrm{A}$ ratio $=0.8$ to 1.5 , DT and IVRT $<90 \mathrm{~ms}$; restrictive pattern defined as the $\mathrm{E} / \mathrm{A}$ ratio $\geq 2$, DT $<140 \mathrm{~ms}$ and IVRT $\leq 70 \mathrm{~ms}$. If necessary, a Valsalva manoeuvre was performed to distinguish between normal and pseudonormal filling. ${ }^{21}$ Tissue-Doppler derived mitral annular peak early velocity $\left(\mathrm{e}^{\prime}\right)$ at the septal and lateral mitral annulus was acquired, and septal, lateral and averaged $\mathrm{E} / \mathrm{e}^{\prime}$ ratios were calculated, respectively. Diastolic dysfunction was further graded by an integral approach based on the assessment of diastolic filling pattern, $\mathrm{E} / \mathrm{e}^{\prime}$, and left atrial volume together with tricuspid regurgitation velocity. ${ }^{21}$

\section{Speckle-tracking imaging}

STI analysis was performed using EchoPAC software (GE, Horten, Norway) as described previously. ${ }^{20}$ Longitudinal peak systolic strain rate (LSr_sys) and LS_sys, longitudinal early (LSr_E) and late (LSr_A) diastolic peak strain rates of each segment were measured. Global deformation values were acquired by averaging strain rate and strain values of all 16 segments. The ratio of $\mathrm{E}$ to global LSr_E (E/LSr_E) was calculated. Images and loops were analysed by an experienced echocardiographer (DL) with $>6$ years of experience in STI analysis, who was blinded to all patient information, including the final histopathological diagnosis.

\section{Renal function}

Glomerular filtration rate (GFR) was measured using technetium 99m-diethylene triamine penta-acetic acid aerosol clearance (Tc99m-DTPA) and estimated 
according to the Modification of Diet in Renal Disease formula.

\section{Data analysis}

Continuous variables were expressed as mean $\pm \mathrm{SD}$ or median (quartiles). Categorical variables were presented as count (per cent). Differences on continuous data with normal distribution across groups were compared using unpaired Student 's t-test. Variables with skewed distribution were tested by Mann-Whitney U-test. Categorical data were compared using a similar approach employing ${ }^{2}$ and Fisher's exact test, as appropriate. Multivariable logistic regression was conducted to define the independent determinants for LE after adjustment for age, sex, body mass index, NYHA functional class and GFR. Receiver operating characteristic (ROC) analysis was performed to identify the diagnostic performance of markers for LE. For the determination of the cut-off values of septal $\mathrm{E} / \mathrm{e}^{\prime}$, we used the cut-off value with maximum sensitivity $(<7)$ to rule out the presence of LE, and the cut-off value with maximum specificity (>14.8) to predict the presence of LE. The respective cut-off values were determined by maximising sensitivity and specificity. Statistical significance was defined as $\mathrm{p}<0.05$ (two-tailed test). Statistical analysis was performed using IBM SPSS, version 23 for Windows (IBM, New York, USA).

\section{RESULTS}

\section{Baseline clinical characteristics}

Clinical characteristics of patients with FD are summarised in table 1 . LE was detected by cMRI in 57 out of 146 (39\%) patients with FD with preserved LVEF. Patients were divided into two groups with and without LE. The mean age was $39 \pm 14$ years, and $57(39 \%)$ patients were men. Patients with LE were older than those without LE $(48 \pm 11$ vs $33 \pm 12$ years, $\mathrm{p}<0.001)$. The percentage of patients with NYHA functional class III-IV was significantly higher in the LE group than in the no LE group $(14.1 \%$ vs $2.2 \%, \mathrm{p}<0.001)$. Prevalence of kidney dysfunction and levels of serum $\mathrm{N}$-terminal pro-brain natriuretic peptide (NT-proBNP) were significantly higher in the LE group than in the no LE group (both $\mathrm{p}<0.05$ ).

\section{Cardiac MRI}

Mean LVEF as measured by cMRI was $64 \% \pm 8 \%$, and similar between groups (table 2). LV mass index was higher in the LE group than in the no LE group $(95 \pm 32$ vs $\left.71 \pm 19 \mathrm{~g} / \mathrm{m}^{2}, \mathrm{p}<0.001\right)$. As shown in online supplementary eFigure $2 \mathrm{~A}$, LE was predominantly detected at basal and mid segments of the inferolateral wall $(82.8 \%$ in segment 5 and $51.6 \%$ in segment 11 ).

\section{Echocardiography}

The mean LVEF derived from echocardiography was $66 \% \pm 6 \%$ and similar between groups (table 3 ). The tricuspid annular plane systolic excursion, a parameter reflecting right ventricular function, ${ }^{22}$ was within the normal range (mean $23.0 \pm 3.9 \mathrm{~mm}$ ) in the total sample. $\mathrm{LV}$ wall thickness, left atrium volume index (LAVI), septal and lateral $\mathrm{E} / \mathrm{e}^{\prime}$ were significantly higher, while septal and lateral $\mathrm{e}^{\prime}$ were significantly lower in the LE group than in the no LE group. The proportion of moderate to severe diastolic dysfunction ${ }^{23}$ was significantly higher in the LE group than the no LE group ( $42.1 \%$ vs $2.1 \%, \mathrm{p}<0.001)$.

\section{Longitudinal systolic and diastolic strain and strain rate}

Global LS_sys, LSr_sys and LSr_E were significantly reduced, and E/LSr_E ratio was significantly increased in the LE group compared with the no LE group, while global LSr_A was similar between groups (table 3). Segmental LS_sys and LSr_E were significantly lower in the LE group than in the no LE group, especially at the mid-lateral segment. In line with the distribution of LE (online supplementary eFigure 2), LS_sys and LSr_E were predominantly lower in the basal and mid-lateral wall (segments 5-6 and 11-12).

\section{Echocardiographic parameters associated with LE}

Associations between clinical and echocardiographic parameters and LE are listed in table 4. NT-proBNP levels, end-diastolic interventricular septal thickness, left ventricular mass indexed to body surface area, LAVI, septal and lateral E/e' ratio, diastolic dysfunction grading, global LS_sys, E/LSr_E, LS_sys and LSr_E at mid-lateral segments were all associated with LE in patients with FD with preserved LVEF after adjustment for age, sex, body mass index, New York Heart Association functional class and Tc99m-DTPA clearance. The ROC analysis showed that septal E/e' was the best echocardiographic marker indicating the presence of LE in patients with FD with preserved LVEF (area under the curve (AUC) 0.86; figure 1). LE was not detected in patients with septal $\mathrm{E} / \mathrm{e}^{\prime}<7$ (sensitivity $100 \%$ and specificity $23.6 \%$ ), while septal $\mathrm{E} / \mathrm{e}^{\prime}>14.8$ was strongly associated with the presence of LE with a specificity of $97.8 \%$ and a sensitivity of $49.1 \%$ (table 5). LE was also detected in 29 out of 116 patients with septal E/e' $\leq 14.8$, indicating there is only a limited negative predictive value to exclude LE using a threshold of $\mathrm{E} / \mathrm{e}^{\prime} \leq 14.8$.

Based on above findings, we established a framework for screening LE of patients with FD using echocardiography in the clinical setting. Figure 2 shows an algorithm employing echocardiographic parameters suggestive of LE in patients with FD with preserved LVEF.

In addition, we defined 45 patients with FD with neither kidney nor cardiac involvement, that is, normal renal function (Tc99m-DTPA clearance $>90 \mathrm{~mL} / \mathrm{min} / 1.73 \mathrm{~m}^{2}$ ), normal NT-proBNP $(<100 \mathrm{pg} / \mathrm{mL})$, normal systolic function (global longitudinal strain $>18 \%$ ), normal LV wall thickness (IVSd $<11 \mathrm{~mm}$ ) and normal LA (LAVI $<34 \mathrm{~mL}$ ) $\mathrm{m}^{2}$ ). Local LE at basal inferior and lateral wall was still evidenced in four $(8.9 \%)$ patients, indicating LE can be present even in the absence of measurable cardiac/ renal dysfunction in patients with FD. On the other hand, LE was not detected in $41.9 \%$ of $(18 / 43)$ patients with 
Table 1 Baseline clinical characteristics

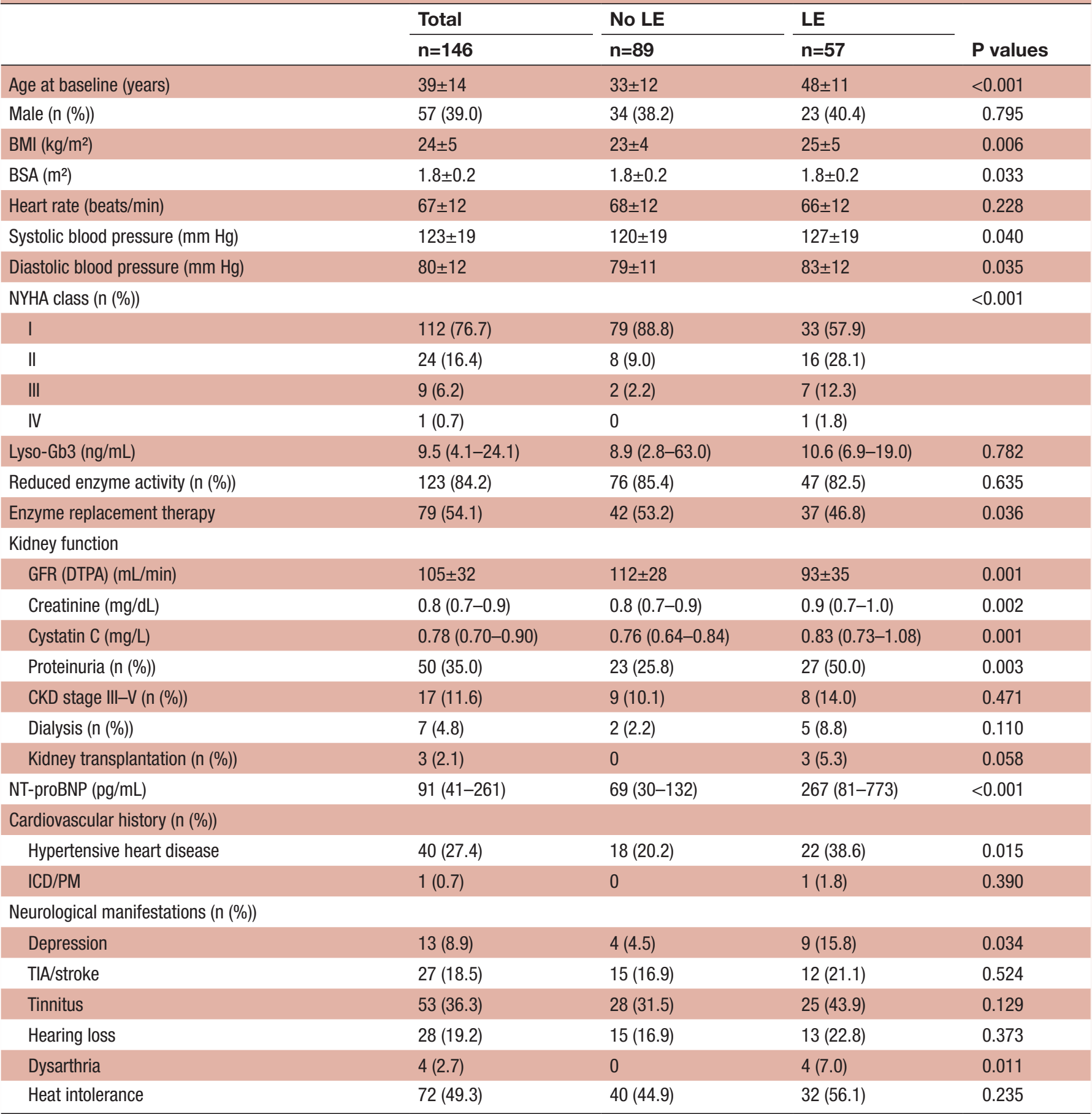

Continuous variables are presented as mean \pm SD or median (quartiles), and categorical variables are presented with counts and percentages. $P<0.05$ vs no LE group.

BMI, body mass index; BSA, body surface area; CKD, chronic kidney disease; GFR (DTPA): glomerular filtration rate from 99mTc-diethylene triamine penta-acetic acid renography; ICD, implantable cardioverter defibrillator; LE, late gadolinium enhancement; Lyso-Gb3: plasma globotriaosylsphingosine; NT-proBNP, N-terminal pro-brain natriuretic peptide; NYHA, New York Heart Association; PCl, percutaneous coronary intervention; PM, pacemaker; TIA, transient ischaemic attack.

a Tc99m-DTPA clearance of $60-90 \mathrm{~mL} / \mathrm{min} / 1.73 \mathrm{~m}^{2}$ (mild renal dysfunction) and in $41.2 \%(7 / 17)$ of patients with a Tc99m-DTPA clearance of $<60 \mathrm{~mL} / \mathrm{min} / 1.73 \mathrm{~m}^{2}$ (moderate to severe renal dysfunction), reflecting vast pathological variance of FD. ${ }^{424}$ 
Table 2 Cardiac MRI characteristics

\begin{tabular}{|c|c|c|c|c|}
\hline & Total & No LE & LE & \multirow[b]{2}{*}{$P$ values } \\
\hline & $n=146$ & $n=89$ & $\mathrm{n}=57$ & \\
\hline LVEF (\%) & $64 \pm 8$ & $62 \pm 7$ & $66 \pm 7$ & 0.004 \\
\hline LVMI $\left(\mathrm{g} / \mathrm{m}^{2}\right)$ & $80 \pm 28$ & $71 \pm 19$ & $95 \pm 32$ & $<0.001$ \\
\hline EDVI $\left(\mathrm{mL} / \mathrm{m}^{2}\right)$ & $76 \pm 18$ & $77 \pm 15$ & $74 \pm 21$ & 0.281 \\
\hline ESVI $\left(\mathrm{mL} / \mathrm{m}^{2}\right)$ & $28 \pm 10$ & $29 \pm 9$ & $25 \pm 11$ & 0.037 \\
\hline SVI $\left(m L / m^{2}\right)$ & $48 \pm 12$ & $48 \pm 10$ & $47 \pm 15$ & 0.779 \\
\hline $\mathrm{Cl}\left(\mathrm{L} / \mathrm{min} / \mathrm{m}^{2}\right)$ & $3.2 \pm 0.8$ & $3.3 \pm 0.8$ & $3.1 \pm 0.9$ & 0.073 \\
\hline
\end{tabular}

Continuous variables are presented as mean \pm SD.

$\mathrm{P}<0.05$ vs no LE group.

$\mathrm{Cl}$, cardiac index; EDVI, end-diastolic volume indexed to body surface area; ESVI, end-systolic volume indexed to body surface area; LE, late gadolinium enhancement; LVEF, left ventricular ejection fraction; LVMI, left ventricular mass indexed to body surface area; SVI, stroke volume indexed to body surface area.

systolic dysfunction, hypertrophy and myocardial fibrosis remain elusive. To the best of our knowledge, this is the first report focusing on the association of both classical and modern diastolic/systolic indices with the presence of myocardial replacement fibrosis in patients with FD with preserved LVEF. The major findings of the present study in patients with FD with preserved LVEF are (1) LE is significantly associated with age, kidney function, NT-proBNP, LV hypertrophy, and systolic and diastolic dysfunction; (2) septal E/ $\mathrm{e}^{\prime}$ ratio appears to be the best echocardiographic marker associated with LE; (3) septal E/e'>14.8 is strongly associated with the presence of LE and (4) LE can sometimes be detected in FD in the absence of additional measurable cardiac/renal dysfunction.

\section{LE in patients with FD with preserved LVEF}

The appearance of myocardial replacement fibrosis is an acknowledged key feature in the cardiac pathophysiology of FD. ${ }^{5}$ It has been postulated that tissue ischaemia secondary to endothelial accumulation of glycosphingolipids in the microvasculature might accelerate the development of myocardial fibrosis in FD, with the inferolateral wall representing the predominantly affected segment. ${ }^{525}$ Previous cMRI studies from our group found that LE was present in $33 \%$ of female and $48 \%$ of male patients with FD. ${ }^{26}$ In line with previous findings from others as well as our own group, ${ }^{19}$ we here confirmed that LE mostly presented at basal and mid-segments of the inferolateral wall, and mid-lateral LS_sys was often significantly reduced despite preserved global LS_sys (figure 1). However, a recent biopsy study revealed that interstitial myocardial fibrosis was already detectable at very early stages of the Fabry cardiomyopathy. ${ }^{27}$ Accordingly, the current study confirmed that LE can be detected at least in some patients with FD in the absence of measurable cardiac and renal dysfunction, suggesting that LE may appear at very early stages of the disease and is neither necessarily related to prior larger-scale morphological nor functional impairments. Pathophysiological explanation might be provided in a current study using cMRI T2 mapping, showing that (chronic) inflammation might contribute to the development of $\mathrm{LE}$ in $\mathrm{FD} .^{28} \mathrm{In}$ line with these findings, we could just recently show that LE is not necessarily progressive, but might even regress slightly under appropriate therapy. ${ }^{29}$ These observations might not be specific or limited to FD, since similar phenomena were evidenced recently also in patients with aortic stenosis undergoing aortic valve replacement. ${ }^{30}$

\section{Association of systolic and diastolic dysfunction with LE}

Progressive LV hypertrophy, diastolic dysfunction and preserved systolic function have been described as the major echocardiographic features of Fabry cardiomyopathy. Pieroni et al demonstrated that diastolic dysfunction can already exist prior to the development of LV hypertrophy or systolic dysfunction in patients with Fabry cardiomyopathy, suggesting that diastolic dysfunction might be an early sign of cardiac involvement in patients with FD. ${ }^{31}$ In line with these findings, we found that diastolic dysfunction was already present in $19 \%(17 / 88)$ of patients with FD with normal LS_sys $(>18 \%)$ and normal LVEF in the current patient cohort.

Previous studies also indicated that the tissue Doppler derived diastolic index (ie, $\mathrm{e}^{\prime}$ ) could provide satisfactory preclinical evidence for diastolic dysfunction in patients with Fabry cardiomyopathy. ${ }^{31}$ Shanks et al further showed that STI-derived longitudinal systolic strain and diastolic isovolumetric strain rate were superior to other classic echocardiographic parameters for the risk stratification and disease staging in patients with FD. ${ }^{32}$ Our results indicate that despite a preserved systolic function (LVEF: mean value $66 \%$, range $52 \%$ to $80 \%$ ), a significant proportion of patients with FD $(35 \%)$ presented with at least mild diastolic dysfunction, as shown by abnormal conventional and STI-derived diastolic deformation indices (LSr_E and E/LSr_E). Moreover, increased LV wall thickness, left atrium volume, septal E/e', E/LSr_E and reduced septal and lateral $\mathrm{e}^{\prime}$ were evidenced in the LE group compared with the no LE group, reflecting that diastolic dysfunction is closely linked to LE. Our results further revealed that serum and echocardiographic biomarkers reflecting diastolic function (including the myocardial stress biomarker NT-proBNP, LV wall thickness, LA volume, $\mathrm{e}^{\prime}$ and $\mathrm{E} / \mathrm{e}^{\prime}$ ) remained predictive of LE, supporting the close relationship between diastolic dysfunction and replacement myocardial fibrosis in FD. However, the STI-derived diastolic index was not superior to the conventional echocardiographic diastolic index. Indeed, our results showed that $\mathrm{e}^{\prime}$ and $\mathrm{E} / \mathrm{e}^{\prime}$ were the best echocardiographic markers suggestive of LE in FD, superior to $\mathrm{E}$ wave, E/A, and DT, and STI-derived global LSr_E and E/LSr_E.

A recent study demonstrated that $\mathrm{E} / \mathrm{e}^{\prime}$ was associated with myocardial fibrosis detected by cMRI in children with hypertrophic cardiomyopathy. ${ }^{33}$ In line with these findings, our data showed a close correlation between $\mathrm{E} / \mathrm{e}^{\prime}$ and LE in patients with FD and preserved LVEF. Septal 
Table 3 Echocardiographic characteristics

\begin{tabular}{|c|c|c|}
\hline Total & No LE & LE \\
\hline$n=146$ & $\mathrm{n}=89$ & $n=57$ \\
\hline
\end{tabular}

\section{Standard echocardiography}

Cardiac dimensions

\begin{tabular}{|c|c|c|c|c|}
\hline LVEDD (mm) & $47 \pm 5$ & $47 \pm 5$ & $46 \pm 6$ & 0.150 \\
\hline LVESD (mm) & $29 \pm 4$ & $30 \pm 4$ & $28 \pm 5$ & 0.012 \\
\hline IVSd (mm) & $10.0 \pm 2.4$ & $9.0 \pm 1.5$ & $11.7 \pm 2.6$ & $<0.001$ \\
\hline LVPWd (mm) & $9.9 \pm 2.4$ & $8.9 \pm 1.7$ & $11.5 \pm 2.5$ & $<0.001$ \\
\hline LVMI $\left(g / \mathrm{m}^{2}\right)$ & $92 \pm 30$ & $82 \pm 23$ & $108 \pm 32$ & $<0.001$ \\
\hline RWT & $0.43 \pm 0.13$ & $0.38 \pm 0.08$ & $0.51 \pm 0.15$ & $<0.001$ \\
\hline RVD_middle (mm) & $27 \pm 4$ & $27 \pm 5$ & $26 \pm 5$ & 0.478 \\
\hline $\mathrm{RAA}\left(\mathrm{cm}^{2}\right)$ & $14 \pm 3$ & $14 \pm 3$ & $14 \pm 3$ & 0.458 \\
\hline LAVI $\left(\mathrm{mL} / \mathrm{m}^{2}\right)$ & $24 \pm 9$ & $21 \pm 6$ & $28 \pm 11$ & $<0.001$ \\
\hline \multicolumn{5}{|l|}{ RV function } \\
\hline TAPSE (mm) & $23.0 \pm 3.9$ & $23.8 \pm 3.7$ & $21.8 \pm 4.0$ & 0.002 \\
\hline sPAP (mm Hg) & $26 \pm 7$ & $25 \pm 5$ & $27 \pm 9$ & 0.136 \\
\hline \multicolumn{5}{|l|}{ LV systolic function } \\
\hline LVEF (\%) & $66 \pm 6$ & $65 \pm 6$ & $68 \pm 6$ & 0.007 \\
\hline \multicolumn{5}{|l|}{ LV diastolic function } \\
\hline E wave (cm/s) & $87 \pm 19$ & $86 \pm 16$ & $89 \pm 21$ & 0.343 \\
\hline E/A ratio & $1.47 \pm 0.54$ & $1.5 \pm 0.5$ & $1.4 \pm 0.6$ & 0.653 \\
\hline DT (ms) & $207 \pm 50$ & $203 \pm 43$ & $214 \pm 58$ & 0.173 \\
\hline IVRT (ms) & $89 \pm 24$ & $79 \pm 17$ & $104 \pm 27$ & $<0.001$ \\
\hline Septal $e^{\prime}(\mathrm{cm} / \mathrm{s})$ & $8.6 \pm 3.3$ & $10.1 \pm 2.9$ & $6.3 \pm 2.3$ & $<0.001$ \\
\hline Septal E/e' ratio & $11.7 \pm 5.8$ & $9.1 \pm 3.1$ & $15.8 \pm 6.7$ & $<0.001$ \\
\hline Lateral $\mathrm{e}^{\prime}(\mathrm{cm} / \mathrm{s})$ & $11.9 \pm 4.2$ & $13.7 \pm 3.8$ & $9.2 \pm 3.1$ & $<0.001$ \\
\hline Lateral E/e' ratio & $8.3 \pm 3.7$ & $6.7 \pm 2.0$ & $10.7 \pm 4.3$ & $<0.001$ \\
\hline Diastolic dysfunction & & & & $<0.001$ \\
\hline Normal & 95 (65.1) & $76(85.4)$ & $19(33.3)$ & \\
\hline Mild & $24(16.4)$ & $10(11.2)$ & $14(24.6)$ & \\
\hline Moderate & $19(13.0)$ & $3(2.1)$ & $16(28.1)$ & \\
\hline Severe & $8(5.5)$ & 0 & $8(14.0)$ & \\
\hline \multicolumn{5}{|c|}{ Speckle-tracking imaging (18 segments averaged) } \\
\hline LS_sys (\%) & $-17.9 \pm 3.3$ & $-18.9 \pm 2.5$ & $-16.3 \pm 3.7$ & $<0.001$ \\
\hline LSr_sys $\left(\mathrm{s}^{-1}\right)$ & $-0.99 \pm 0.16$ & $-1.03 \pm 0.15$ & $-0.94 \pm 0.17$ & 0.001 \\
\hline $\mathrm{LSr} \_\mathrm{E}\left(\mathrm{s}^{-1}\right)$ & $1.36 \pm 0.41$ & $1.49 \pm 0.39$ & $1.16 \pm 0.36$ & $<0.001$ \\
\hline $\mathrm{LSr} \_\mathrm{A}\left(\mathrm{S}^{-1}\right)$ & $0.66 \pm 0.23$ & $0.66 \pm 0.22$ & $0.65 \pm 0.25$ & 0.938 \\
\hline E/LSr_E (cm) & $69.0 \pm 25.3$ & $60.5 \pm 16.3$ & $82.6 \pm 30.8$ & $<0.001$ \\
\hline
\end{tabular}

Continuous variables are presented as mean $\pm \mathrm{SD}$, and categorical variables are presented with counts and percentages.

$\mathrm{P}<0.05$ vs no LE group.

DT, deceleration time of $E$ wave; $E$ wave, mitral inflow early diastolic filling velocity; $E / A$ ratio, the ratio of mitral inflow early filling velocity to late diastolic filling velocity; E/e', the ratio of early diastolic mitral inflow velocity to mitral annular tissue velocity; E/LSr_E, the ratio of mitral inflow early diastolic filling velocity to LSr_E; e', tissue Doppler-derived mitral annular early diastolic velocity; IVRT, isovolumetric relaxation time; IVSd, end-diastolic interventricular septal thickness; LAVI, left atrial volume indexed to body surface area; LE, late gadolinium enhancement; LS_sys, longitudinal systolic strain; LSr_A, longitudinal late diastolic strain rate; LSr_E, longitudinal early diastolic strain rate; LSr_sys, longitudinal systolic strain rate; LVEDD, left ventricular end-diastolic dimension; LVEF, left ventricular ejection fraction; LVESD, left ventricular end-systolic dimension; LVMI, left ventricular mass indexed to body surface area; LVPWd, end-diastolic posterior wall thickness; RAA, end-systolic right atrial area; RV, right ventricle; RVD, end-diastolic mid-right ventricular diameter; RWT, relative wall thickness sPAP, systolic pulmonary artery pressure; TAPSE, tricuspid annular plane systolic excursion. 


\begin{tabular}{|c|c|c|c|c|c|c|c|c|}
\hline & Spearman's $r$ & $P$ values & OR $^{*}$ & $95 \% \mathrm{Cl}$ & $P$ values & AUC & $95 \% \mathrm{Cl}$ & $P$ values \\
\hline \multicolumn{9}{|l|}{ Clinical marker } \\
\hline NT-proBNP (pg/mL)† & 0.47 & $<0.001$ & 1.85 & 1.18 to 2.92 & 0.008 & 0.78 & 0.70 to 0.85 & $<0.001$ \\
\hline \multicolumn{9}{|l|}{ Echocardiographic markers } \\
\hline IVSd (mm) & 0.55 & $<0.001$ & 1.65 & 1.25 to 2.18 & $<0.001$ & 0.82 & 0.75 to 0.89 & $<0.001$ \\
\hline $\operatorname{LVMI}\left(\mathrm{g} / \mathrm{m}^{2}\right)$ & 0.42 & $<0.001$ & 1.03 & 1.01 to 1.05 & 0.002 & 0.75 & 0.66 to 0.83 & $<0.001$ \\
\hline $\mathrm{EF}(\%)$ & 0.23 & 0.006 & 1.05 & 0.97 to 1.13 & 0.222 & 0.63 & 0.54 to 0.73 & 0.006 \\
\hline LAVI (mL) & 0.39 & $<0.001$ & 1.10 & 1.03 to 1.18 & 0.004 & 0.73 & 0.64 to 0.82 & $<0.001$ \\
\hline Septal E/e' & 0.60 & $<0.001$ & 1.30 & 1.13 to 1.49 & $<0.001$ & 0.86 & 0.79 to 0.92 & $<0.001$ \\
\hline Lateral E/e' & 0.54 & $<0.001$ & 1.34 & 1.11 to 1.62 & 0.002 & 0.82 & 0.75 to 0.89 & $<0.001$ \\
\hline Diastolic dysfunction grading & 0.56 & $<0.001$ & 2.98 & 1.61 to 5.526 & 0.001 & 0.78 & 0.70 to 0.86 & $<0.001$ \\
\hline \multicolumn{9}{|l|}{ STI markers } \\
\hline Global LS_sys (\%) & 0.38 & $<0.001$ & 1.16 & 1.00 to 1.34 & 0.047 & 0.73 & 0.65 to 0.81 & $<0.001$ \\
\hline E/LSr_E (cm) & 0.42 & $<0.001$ & 1.03 & 1.00 to 1.05 & 0.015 & 0.74 & 0.65 to 0.82 & $<0.001$ \\
\hline Mid-lateral LS_sys (\%) & 0.51 & $<0.001$ & 1.20 & 1.07 to 1.34 & 0.002 & 0.83 & 0.75 to 0.90 & $<0.001$ \\
\hline Mid-lateral LSr_E $\left(\mathrm{s}^{-1}\right)$ & -0.44 & $<0.001$ & 0.38 & 0.15 to 0.95 & 0.034 & 0.76 & 0.68 to 0.84 & $<0.001$ \\
\hline
\end{tabular}

*Adjusted for age, sex, BMI, NYHA functional class, GFR (DTPA).

†Ln transformed.

AUC, area under the curve; BMI, body mass index; E/e', the ratio of early diastolic mitral inflow velocity to mitral annular tissue velocity; EF, ejection fraction; GFR (DTPA), glomerular filtration rate from 99mTc-diethylene triamine penta-acetic acid; IVSd, end-diastolic interventricular septal thickness; LAVI, left atrial volume indexed to body surface area; LE, late gadolinium enhancement; LS_sys, longitudinal systolic strain; LSr_E, longitudinal early diastolic strain rate; LVEF, left ventricular ejection fraction; LVMI, left ventricular mass indexed to body surface area; NT-proBNP, N-terminal pro-brain natriuretic peptide; NYHA, New York Heart Association; STI, speckle-tracking imaging.

$\mathrm{E} / \mathrm{e}^{\prime}<7$ indicated absence of LE in patients with FD. On the other hand, LE was detected in $93 \%$ of patients with septal E/e'>14.8. In patients presenting with mid-ranged septal E/e' (7-14.8), an increased lateral E/e' $(>13)$ might provide additional information suggestive of LE.

\section{Limitations}

The patient sample was relatively small in this retrospective study, which could have introduced overoptimism regarding the proposed cut-off values of index test. Future prospective studies are warranted to verify the clinical value of the proposed algorithm, in particular as segmental STI has technical limitations specifically regarding interobserver and intraobserver variability. In our algorithm, we used reduced segmental longitudinal strain/strain rate at mid-lateral wall (ie, LS_midLat and LSrE_midLat) as one screening criteria suggestive of LE, since related changes in this segment belong to pathological feature of cardiac involvement of FD. Of note, however, previous studies showed that single segmental strain values are associated with higher variability in contrast to global longitudinal strain. ${ }^{34}$ Additionally, potential misclassification in some groups of patients with 'grey zone' E/e' could be as high as $20 \%$ according to our proposed algorithm. This scenario further implies the importance of cMRI examination in patients with FD with preserved LVEF.

Even though the presence of myocardial fibrosis can influence the management of patients with FD, therapeutic approaches might be affected not only by the presence, but more importantly the absolute amount of LE. In the current study, we only investigated the presence or absence of LE and the severity of LE was not analysed. This study limitation particularly needs to be clarified in future studies aiming to observe the clinical outcome of patients with FD with various degrees of LE severity and therapy regimens.

\section{Clinical implications}

Both echocardiography and MRI examinations are valuable tools for monitoring the cardiac involvement in patients with FD. Even though a close correlation of LE with diastolic functional parameters was found, the current results also show that LE can be detected even in the absence of measurable cardiac involvement at least in some patients with FD. It has been reported that ERT can reduce glycosphingolipid accumulation in the kidney of patients with FD and even clean intracellular deposits. ${ }^{35}$ ERT also improves outcome, however, is presumably less effective in patients with FD who already developed myocardial fibrosis. ${ }^{17} 27$ As a result, our study results strongly support additional routine examinations both by echocardiography as well as by cMRI in patients with FD at risk of organ involvement in order to facilitate a therapy start as early as possible to avoid permanent organ damage. In patients not feasible to undergo MRI, diastolic function as acquired by echocardiography might additionally be used as a surrogate 

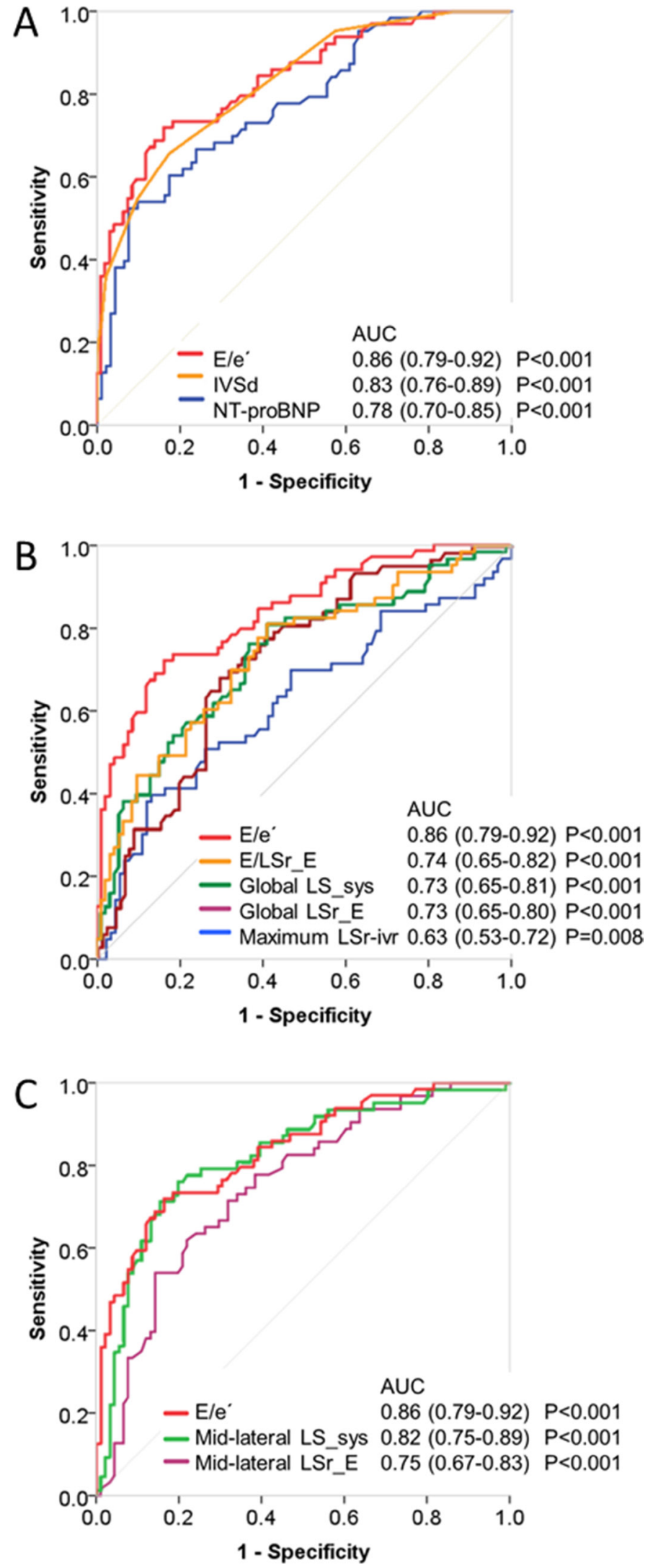

Figure 1 Receiver operating characteristic analyses of NT-proBNP and echocardiographic determinants for late gadolinium enhancement (LE) in patients with Fabry disease with preserved left ventricular ejection fraction. AUC, area under curve; E/e', the ratio of early diastolic mitral inflow velocity to mitral annular tissue velocity; IVSd, end-diastolic interventricular septal thickness; NT-proBNP, N-terminal pro-brain natriuretic peptide; LS_sys, longitudinal systolic strain; LSr_E, longitudinal early diastolic strain rate; E/LSr_E, the ratio of mitral inflow early diastolic filling velocity to LSr_E; LSr_ivr, longitudinal peak strain rate during isovolumetric relaxation time. 
Table 5 Diagnostic performance of septal E/e' for LE in patients with Fabry disease

\begin{tabular}{|c|c|c|c|c|c|}
\hline $\begin{array}{l}\text { No LE } \\
(n=89)\end{array}$ & $\begin{array}{l}\text { LE } \\
(n=57)\end{array}$ & Sensitivity $(95 \% \mathrm{Cl})$ & $\begin{array}{l}\text { Specificity } \\
\text { (95\% Cl) }\end{array}$ & PPV (95\% Cl) & NPV $(95 \% \mathrm{Cl})$ \\
\hline
\end{tabular}

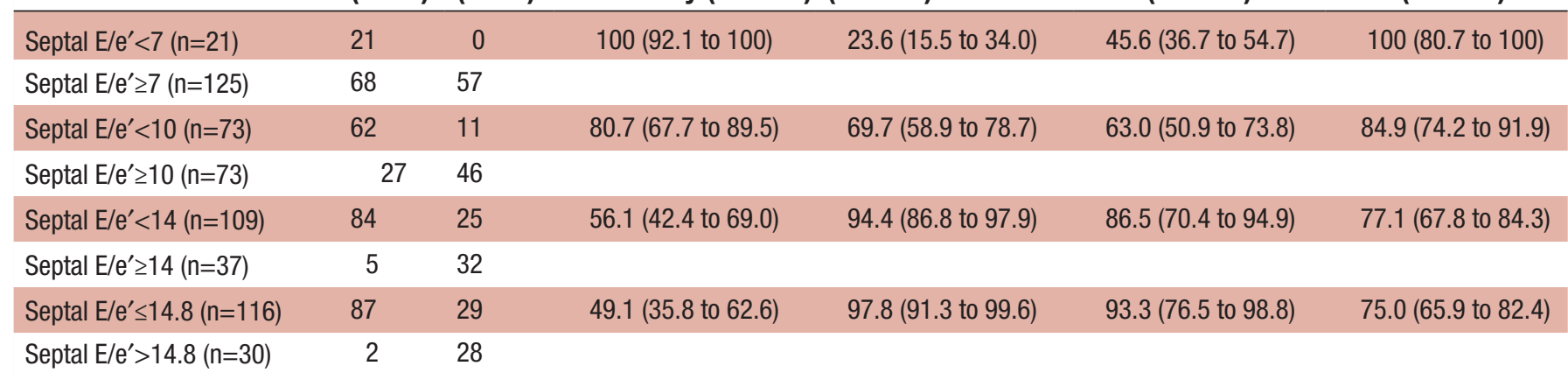

E/e', the ratio of early diastolic mitral inflow velocity to mitral annular tissue velocity; LE, late gadolinium enhancement; PPV, positive predictive value; NPV, negative predictive value.

measure to predict presence and evolution of replacement fibrosis.

\section{CONCLUSIONS}

LE is significantly associated with age, kidney function, NT-proBNP, LV hypertrophy, and systolic and diastolic dysfunction in patients with FD with preserved LVEF. In these patients, septal $\mathrm{E} / \mathrm{e}^{\prime}$ ratio is the best echocardiographic marker associated with prevalence of LE. However, diastolic dysfunction is not a prerequisite for LE in FD, since LE can precede measurable functional impairments as very early marker of cardiac involvement.

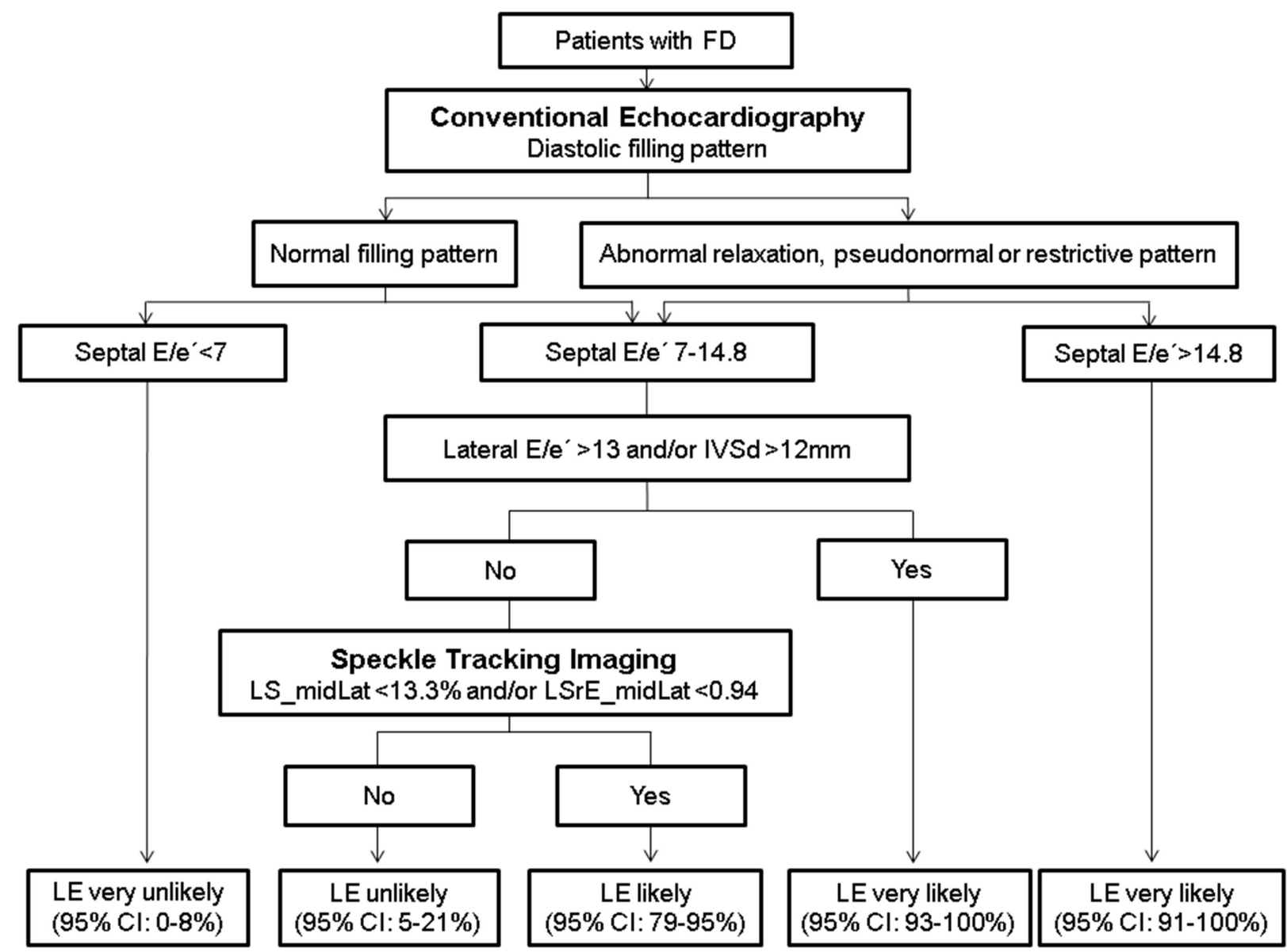

Figure 2 Screening algorithm by the use of echocardiographic parameters suggestive of late gadolinium enhancement (LE) in patients with Fabry disease with preserved left ventricular ejection fraction. $95 \% \mathrm{Cl}$ for proportions was calculated according to the efficient-score method (corrected for continuity) described by R. Newcombe, based on the procedure outlined by $\mathrm{E}$. B. Wilson in 1927. E/e', the ratio of early diastolic mitral inflow velocity to mitral annular tissue velocity; IVSd, end-diastolic interventricular septal thickness; LSr_E, longitudinal early diastolic strain rate. 
Contributors Conception and design: DL, KH, PN. Patient inclusion: D0, TS, JM. Collection and assembly of data: DL, DO, TS, KH, JM, FW, SH. Data analysis and interpretation: DL, KH, SS, PN. Administrative support: GE, CW, SF, PN. Manuscript drafting: DL, KH, PN. Revisions and final manuscript approval: All authors . DL, KH and PN serve as guarantors responsible for the overall content of the manuscript.

Funding This work was supported by grants from the Bundesministerium für Bildung und Forschung (BMBF 01E01504 M0.2 and M0.6) and Genzyme.

Competing interests PN, FW and CW have received speaker and/or advisory board honoraria from Amicus, Genzyme and Shire. Research grants were given to the institution by Genzyme and Shire.

Patient consent Not required.

Ethics approval The local ethics committee at the University of Würzburg.

Provenance and peer review Not commissioned; externally peer reviewed.

Data sharing statement All available data can be obtained from the corresponding author.

Open access This is an open access article distributed in accordance with the Creative Commons Attribution Non Commercial (CC BY-NC 4.0) license, which permits others to distribute, remix, adapt, build upon this work non-commercially, and license their derivative works on different terms, provided the original work is properly cited, appropriate credit is given, any changes made indicated, and the use is non-commercial. See: http://creativecommons.org/licenses/by-nc/4.0/.

\section{REFERENCES}

1. Desnick RJ, Brady R, Barranger J, et al. Fabry disease, an underrecognized multisystemic disorder: expert recommendations for diagnosis, management, and enzyme replacement therapy. Ann Intern Med 2003;138:338-46.

2. Weidemann F, Niemann M, Störk S, et al. Long-term outcome of enzyme-replacement therapy in advanced Fabry disease: evidence for disease progression towards serious complications. J Intern Med 2013;274:331-41.

3. Mehta A, Clarke JT, Giugliani R, et al. Natural course of Fabry disease: changing pattern of causes of death in FOS - Fabry Outcome Survey. J Med Genet 2009;46:548-52.

4. Oder D, Liu D, Hu K, et al. $\alpha$-Galactosidase a genotype N215S Induces a specific cardiac variant of fabry disease. Circ Cardiovasc Genet 2017;10:e001691.

5. Moon JC, Sachdev B, Elkington AG, et al. Gadolinium enhanced cardiovascular magnetic resonance in Anderson-Fabry disease. Evidence for a disease specific abnormality of the myocardial interstitium. Eur Heart J 2003;24:2151-5.

6. Weidemann F, Linhart A, Monserrat L, et al. Cardiac challenges in patients with Fabry disease. Int J Cardiol 2010;141:3-10.

7. Niemann M, Liu D, Hu K, et al. Prominent papillary muscles in Fabry disease: a diagnostic marker? Ultrasound Med Biol 2011;37:37-43.

8. Seydelmann N, Liu D, Krämer J, et al. High-sensitivity troponin: a clinical blood biomarker for staging cardiomyopathy in fabry disease. J Am Heart Assoc 2016;5:e002839.

9. Pagano JJ, Chow K, Khan A, et al. Reduced right ventricular native myocardial $\mathrm{t} 1$ in Anderson-Fabry Disease: Comparison to Pulmonary Hypertension and Healthy Controls. PLoS One 2016;11:e0157565.

10. Weidemann F, Maier SK, Störk S, et al. Usefulness of an implantable loop recorder to detect clinically relevant arrhythmias in patients with advanced Fabry cardiomyopathy. Am J Cardiol 2016;118:264-74.

11. Mewton N, Liu CY, Croisille P, et al. Assessment of myocardial fibrosis with cardiovascular magnetic resonance. J Am Coll Cardiol 2011;57:891-903

12. Biernacka A, Frangogiannis NG. Aging and cardiac fibrosis. Aging Dis 2011;2:158-73.

13. Martos R, Baugh J, Ledwidge M, et al. Diastolic heart failure: evidence of increased myocardial collagen turnover linked to diastolic dysfunction. Circulation 2007;115:888-95.

14. Moreo A, Ambrosio G, De Chiara B, et al. Influence of myocardial fibrosis on left ventricular diastolic function: noninvasive assessment by cardiac magnetic resonance and echo. Circ Cardiovasc Imaging 2009;2:437-43.
15. Fan D, Takawale A, Lee J, et al. Cardiac fibroblasts, fibrosis and extracellular matrix remodeling in heart disease. Fibrogenesis Tissue Repair 2012;5:15.

16. Ersbøll M, Andersen MJ, Valeur $\mathrm{N}$, et al. Early diastolic strain rate in relation to systolic and diastolic function and prognosis in acute myocardial infarction: a two-dimensional speckle-tracking study. Eur Heart J 2014;35:648-56.

17. Weidemann F, Niemann M, Breunig F, et al. Long-term effects of enzyme replacement therapy on fabry cardiomyopathy: evidence for a better outcome with early treatment. Circulation 2009;119:524-9.

18. Germain DP, Charrow J, Desnick RJ, et al. Ten-year outcome of enzyme replacement therapy with agalsidase beta in patients with Fabry disease. J Med Genet 2015;52:353-8.

19. Krämer J, Niemann M, Liu D, et al. Two-dimensional speckle tracking as a non-invasive tool for identification of myocardial fibrosis in Fabry disease. Eur Heart J 2013;34:1587-96.

20. Liu D, Hu K, Störk S, et al. Predictive value of assessing diastolic strain rate on survival in cardiac amyloidosis patients with preserved ejection fraction. PLoS One 2014;9:e115910.

21. Nagueh SF, Smiseth OA, Appleton CP, et al. Recommendations for the Evaluation of Left Ventricular Diastolic Function by Echocardiography: An Update from the American Society of Echocardiography and the European Association of Cardiovascular Imaging. Eur Heart J Cardiovasc Imaging 2016;17:1321-60.

22. Lang RM, Badano LP, Mor-Avi V, et al. Recommendations for cardiac chamber quantification by echocardiography in adults: an update from the American Society of Echocardiography and the European Association of Cardiovascular Imaging. J Am Soc Echocardiogr 2015;28:1-39.

23. Nagueh SF, Appleton CP, Gillebert TC, et al. Recommendations for the evaluation of left ventricular diastolic function by echocardiography. J Am Soc Echocardiogr 2009;22:107-33.

24. Oder D, Üçeyler N, Liu D, et al. Organ manifestations and long-term outcome of Fabry disease in patients with the GLA haplotype D313Y BMJ Open 2016;6:e010422.

25. Moon JC, Sheppard M, Reed E, et al. The histological basis of late gadolinium enhancement cardiovascular magnetic resonance in a patient with Anderson-Fabry disease. J Cardiovasc Magn Reson 2006:8:479-82.

26. Niemann M, Herrmann S, Hu K, et al. Differences in Fabry cardiomyopathy between female and male patients: consequences for diagnostic assessment. JACC Cardiovasc Imaging 2011;4:592-601.

27. Weidemann F, Sanchez-Niño MD, Politei J, et al. Fibrosis: a key feature of Fabry disease with potential therapeutic implications. Orphanet J Rare Dis 2013;8:116.

28. Nordin S, Kozor R, Bulluck H, et al. Cardiac Fabry disease with late gadolinium enhancement is a chronic inflammatory cardiomyopathy. J Am Coll Cardiol 2016;68:1707-8.

29. Müntze J, Salinger T, Gensler D, et al. Treatment of hypertrophic cardiomyopathy caused by cardiospecific variants of Fabry disease with chaperone therapy. Eur Heart J 2018;39:1861-2.

30. Ribeiro HB, Lerakis S, Gilard M, et al. Transcatheter aortic valve replacement in patients with low-flow, low-gradient aortic stenosis: the TOPAS-TAVi registry. J Am Coll Cardiol 2018;71:1297-308.

31. Pieroni M, Chimenti C, Ricci R, et al. Early detection of Fabry cardiomyopathy by tissue Doppler imaging. Circulation 2003;107:1978-84.

32. Shanks M, Thompson RB, Paterson ID, et al. Systolic and diastolic function assessment in fabry disease patients using speckle-tracking imaging and comparison with conventional echocardiographic measurements. J Am Soc Echocardiogr 2013;26:1407-14.

33. Ziółkowska L, Petryka J, Boruc A, et al. Comparison of echocardiography with tissue Doppler imaging and magnetic resonance imaging with delayed enhancement in the assessment of children with hypertrophic cardiomyopathy. Arch Med Sci 2017;13:328-36.

34. Mirea O, Pagourelias ED, Duchenne J, et al. Variability and reproducibility of segmental longitudinal strain measurement: a report from the EACVI-ASE strain standardization task force. JACC Cardiovasc Imaging 2018;11:15-24.

35. Eng CM, Guffon N, Wilcox WR, et al. Safety and efficacy of recombinant human alpha-galactosidase $A$ replacement therapy in Fabry's disease. N Engl J Med 2001;345:9-16. 